\title{
A LYAPUNOV THEORY BASED UPFC CONTROLLER FOR POWER FLOW CONTROL
}

\author{
Ali Zangeneh, Ahad Kazemi, Majid Hajatipour and Shahram Jadid \\ Center of Excellence for Power Systems Automation and Operation \\ Iran University of Science and Technology, Tehran, Iran
}

\begin{abstract}
Unified Power Flow Controller (UPFC) is the most comprehensive multivariable device among the FACTS controllers. According to high importance of power flow control in transmission lines, a new controller is designed based on the Lyapunov theory. The Main goal of this paper is to design a controller which enables a power system to track reference signals precisely and to be robust in the presence of uncertainty of system parameters and disturbances. The performance of the proposed controller is simulated on a two bus test system and compared with a conventional PI controller.
\end{abstract}

Keywords: FACTS, Lyapunov theory, Power flow control, UPFC

\section{INTRODUCTION}

Nowadays the grow of power systems will rely more on increasing capability of existing transmission systems, rather than on building new transmission lines and power stations, for economical and environmental reasons.

The potential benefits with the utilization of flexible ac transmission system (FACTS) devices include reduction of operation and transmission investment costs, increasing system security and reliability, and increasing transfer capabilities in a deregulated environment [1].

Unified Power Flow Controller (UPFC) is the most comprehensive multivariable device among the FACTS controllers [2]. Simultaneous control of multiple power system variables with UPFC possesses enormous difficulties. In addition, the complexity of the UPFC control increases due to the fact that the controlled and the control variables interact with each other.

UPFC is a power electronic based device which can provide a proper control for impedance, phase angle and reactive power of a transmission line [2]. Each converter of a UPFC can independently generate or absorb reactive power. This arrangement enables free flow of active power in either direction between the ac terminals of the two converters [3].

In recent years a number of investigations have been carried out on various capabilities of UPFC such as power flow control, voltage control, transient stability enhancement, oscillation damping and etc. [4-8].

The performance of the control scheme deteriorates in the presence of uncertainties in system parameters. In this paper, a new controller of UPFC based on Lyapunov theory for power flow control is designed which is able to track reference signals precisely and is robust in the presence of uncertainty of system parameters and disturbances.

\section{UPFC model}

The schematic diagram of a UPFC is shown in Fig. 1. It consists of two back-to-back, selfcommutated, voltage source converters connected through a common de link [9].

As it can be seen in Fig. 1, converter1 is coupled to the AC system through a shunt transformer (excitation transformer) and the converter 2 is coupled through a series transformer (boosting transformer). Note that subscripts ' $s$ ' and ' $r$ ' are used to represent sending and receiving end buses respectively. By regulating the series injected voltage $v_{s e}$, the complex power flow $\left(P_{r}+\mathrm{j} Q_{r}\right)$ through the transmission line can be controlled. The complex power injected by the converter 2 $\left(P_{s e}+\mathrm{j} Q_{s e}\right)$ depends on its output voltage and transmission line current. The injected active power $P_{\text {se }}$ of the series converter is taken from the dc link, which is in turn drawn from the AC system through the converter 1 . On the other hand, both converters are capable of absorbing or supplying reactive power independently. The reactive power of the converter 1 can be used to regulate the voltage magnitude of the bus at which the shunt transformer is connected. 


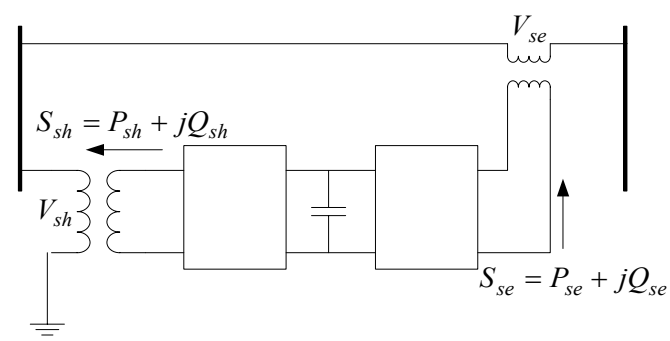

Fig. 1. Schematic diagram of/the UPFC system

The single-phase representation of a three-phase UPFC system is shown in Fig. 2. In this figure both converters are represented by voltage sources $v_{s e}$ and $v_{s h}$, respectively. Also $\left(R=R_{s e}+R_{L}\right)$ and $\left(L=L_{s e}+L_{L}\right)$ represent the resistance and leakage inductance of series transformer and transmission line respectively, similarly $R_{s h}$ afd $L_{s h}$ represent the resistance and leakage inductance of the shunt transformer respectively [9].

The current through the -series and shunt branches of the circuit of Fig. 2 can be expressed by the following differential equations for one phase of the system [9]. These equations can be written for other phases similarly.

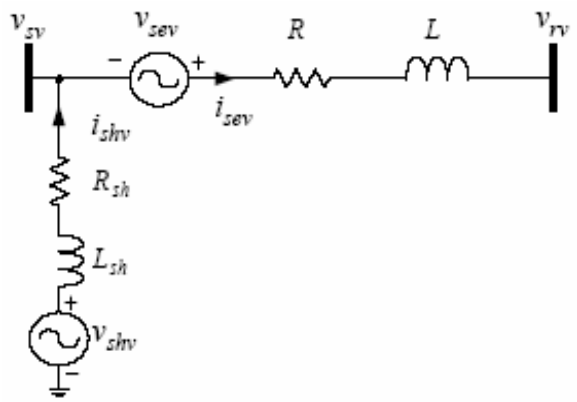

Fig. 2. Single phase representation of the UPFC system

$$
\begin{aligned}
& \frac{d i_{\text {sea }}}{d t}=\frac{1}{L}\left(-R i_{\text {sea }}+v_{\text {sea }}+v_{\text {sa }}-v_{\text {ra }}\right) \\
& \frac{d i_{\text {sha }}}{d t}=\frac{1}{L}\left(-R i_{\text {sha }}+v_{\text {sha }}-v_{\text {sa }}\right)
\end{aligned}
$$

The three-phase system differential equations can be transformed into a " $d, q$ " reference frame using Park's transformation as follows:

$$
\begin{aligned}
& {\left[\begin{array}{l}
i_{\text {sed }} \\
i_{\text {seq }}
\end{array}\right]=\left[\begin{array}{cc}
\frac{-R}{L} & \omega_{b} \\
-\omega_{b} & \frac{-R}{L}
\end{array}\right] \cdot\left[\begin{array}{c}
i_{\text {sed }} \\
i_{\text {seq }}
\end{array}\right]+\frac{1}{L}\left[\begin{array}{c}
v_{\text {sed }}+v_{s d}-v_{r d} \\
v_{\text {seq }}-v_{r q}
\end{array}\right]} \\
& {\left[\begin{array}{l}
i_{\text {shd }} \\
i_{s h q}
\end{array}\right]=\left[\begin{array}{cc}
\frac{-R_{s h}}{L_{s h}} & -\omega_{b} \\
-\omega_{b} & \frac{-R_{s h}}{L_{s h}}
\end{array}\right] \cdot\left[\begin{array}{c}
i_{s h d} \\
i_{s h q}
\end{array}\right]+\frac{1}{L_{s h}}\left[\begin{array}{c}
v_{\text {shd }}+v_{s d} \\
v_{\text {shq }}
\end{array}\right]}
\end{aligned}
$$

where $\omega_{b}=2 \pi f_{b}$, and $f_{b}$ is the fundamental frequency of the supply voltage. Since the Park's transformation used in finding (3) and (4) keeps the instantaneous power invariant and the $d$-axis lies on the space vector of the sending end voltage $v_{s}$, thus $v_{s}=\left(v_{s d}+\mathrm{j} v_{s q}\right)=\left(v_{s d}+\mathrm{j} 0\right)$.

Note that in the above equations, subscripts ' $d$ ' and ' $q$ ' are used to represent the direct and quadrature axes components, respectively $\left(x=x_{d}+\mathrm{j} x_{q}\right)$.

Since the dynamic equations of converter 1 are identical to that of converter 2 as described before, both converters should have identical control strategy. Therefore for the sake of brevity in this paper only the technique of designing the controller of converter 2 is describes in detail.

$$
\begin{array}{ll}
\dot{\mathbf{x}}=\mathbf{A x}+\mathbf{B u}+d & \text { Transformer } \\
\mathbf{y}=\mathbf{C x} &
\end{array}
$$

where $\mathrm{d}$ is the uncertainty vector and state variables equofinkontrol variables vector and output variables vector of converter 2 are defined

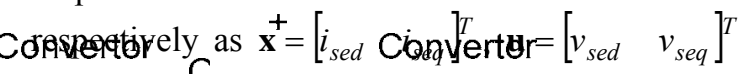

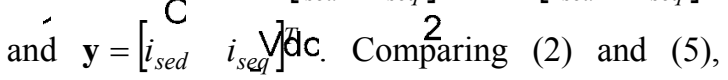
when $v_{s}$ and $v_{r}$ are kept constant, the system matrices $\mathbf{A}, \mathbf{B}$, and $\mathbf{C}$ can be written as:

$\mathbf{A}=\left[\begin{array}{rr}-\frac{R}{L} & \omega_{b} \\ -\omega_{b} & -\frac{R}{L}\end{array}\right] ; \quad \mathbf{B}=\left[\begin{array}{ll}1 & 0 \\ 0 & 1\end{array}\right] ;$
$\mathbf{C}=\left[\begin{array}{ll}1 & 0 \\ 0 & 1\end{array}\right]$

As mentioned previously, the common connection between the two converters is formed by a dc-voltage bus. When the losses in the converters are neglected, the active power balance equation at the dc link can be written as [9]:

$P_{d c}=P_{s e}+P_{s h}$

where $P_{s e}$ and $P_{s h}$ are active power supplied by the converters 1 and 2 respectively which can be obtained as follow:

$P_{\text {se }}=\frac{3}{2}\left(v_{\text {sed }} i_{\text {sed }}+v_{\text {seq }} i_{\text {seq }}\right)$
$P_{\text {sh }}=\frac{3}{2}\left(v_{\text {shd }} i_{\text {shd }}+v_{\text {shq }} i_{\text {shq }}\right)$

Note that, since the power loss of the shunt transformer can be ignored, active power of converter 1 (9) can be written approximately as:

$P_{s h} \approx \frac{3}{2}\left(v_{s d} i_{s h d}\right)$

Also the active power of the dc link is represented as (11):

$P_{d c}=v_{d c} i_{d c}=-C v_{d c} \frac{d v_{d c}}{d t}$

Substituting (11) in (7), equation (12) will be obtained. 
$\frac{d v_{d c}}{d t}=-\frac{1}{C v_{d c}}\left(P_{s e}+P_{s h}\right)$

It is clear that the above equation is non linear, therefore for linearizing and simplifying, the following equation is defined.

$\frac{d v_{d c}^{2}}{d t}=-\frac{2}{C}\left(P_{s e}+P_{s h}\right)$

The following section is assigned to introduce the design of a controller based on the Lyapunov theory. This analysis is based on a simplified mathematical model of the converter connected to a two bus system as shown in Fig. 1.

\section{Design of a controller based on Lyapunov theory}

Fig 3 shows the schematic of a system state space. As it was mentioned, the main goal of this paper is to design a controller which enables the power system to track reference signals precisely and to be robust in the presence of uncertainty of system parameters and disturbances. To reach this purpose a new controller is designed based on the Lyapunov theory in this paper. The controller based on the Lyapunov method is designed as slope changes of energy function which always remains negative $(\dot{V}<0)$ [10]. This energy function consists of a set of error terms. This expression provides stability condition of error terms in the presence of uncertainty and disturbance. Therefore the tracking error and its derivative are defined as below:

$$
\mathbf{e}=\mathbf{x}_{d}-\mathbf{x}
$$

$\dot{\mathbf{e}}=\dot{\mathbf{x}}_{d}-\dot{\mathbf{x}}$

where $\mathbf{x}$ is the vector of state variables and $\mathbf{x}_{d}=\left[\begin{array}{ll}i_{\text {sed }}^{*} & i_{\text {seq }}^{*}\end{array}\right]^{T}$ is the vector of reference signals. In $\mathbf{x}_{\mathrm{d}}$ equation, $i_{\text {sed }}^{*}$ and $i_{\text {seq }}^{*}$ can be obtained similarly by (8) and (9) knowing the active and reactive power references of transmission line $\left(P_{r}^{*}\right.$ and $\left.Q_{r}^{*}\right)$.

$$
\begin{aligned}
& i_{\text {sed }}^{*}=\frac{2}{3} \frac{\left(P_{r}^{*} v_{r d}-Q_{r}^{*} v_{r q}\right)}{\Delta} \\
& i_{\text {seq }}^{*}=\frac{2}{3} \frac{\left(P_{r}^{*} v_{r q}+Q_{r}^{*} v_{r d}\right)}{\Delta}
\end{aligned}
$$

where $\Delta=v_{r d}^{2}+v_{r q}^{2}$.

Substituting (15) in (5), the derivative of tracking (dynamic error) can be obtained:

$$
\dot{\mathbf{e}}=\mathbf{A} \mathbf{e}+\dot{\mathbf{x}}_{d}-\mathbf{A} \mathbf{x}_{d}-\mathbf{d}-\mathbf{B u}
$$

To fulfill stability condition of the system dynamic error, multiplication of control matrix and control variables vector is defined as:

$$
\mathbf{u}^{\prime}=\mathbf{B u}=\mathbf{B k e}+\dot{\mathbf{x}}_{d}-\mathbf{A} \mathbf{x}_{d}-\mathbf{u}_{s}
$$

The control variables vector is calculated by using the inverse of the above equation. The amounts of variables of row matrix $\mathbf{k}$ at equation (19) are set such as the whole eigen values of

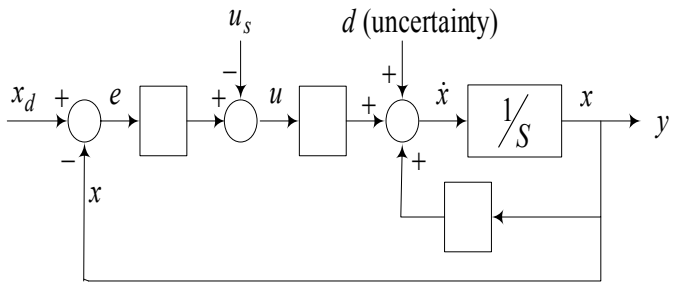

Fig 3. Schematic of system state space

matrix (A-Bk) is lain on the left side of imaginary axis. Vector $\mathbf{u}_{s}$ is also described as an observer signal. The function of this vector is to accomplish stability condition based on Lyapunov theory. Therefore by substitution equation (19) at (18), the new equation is obtained for dynamic error of the system [10].

$\dot{\mathbf{e}}=\mathbf{A}_{0} \mathbf{e}-\mathbf{d}+\mathbf{u}_{s}$

where in the above equation $\mathbf{A}_{0}=\mathbf{A}-\mathbf{B k}$.

To accomplish stability condition, the observer signal is defined as below:

$\mathbf{u}_{s}=-I^{*} \frac{\mathbf{e}^{T} \mathbf{P} \mid}{\mathbf{e}^{T} \mathbf{P}} \cdot d_{m}=-I^{*} \cdot d_{m} \cdot\left|\mathbf{e}^{T} \mathbf{P}\right| \cdot\left(\mathbf{e}^{T} \mathbf{P}\right)^{-1}$

where $I^{*}$ is a positive number $I^{*} \geq 1$.

It is necessary to be noted that $\left(\mathbf{e}^{T} \mathbf{P}\right)$ is not a square matrix and therefore pseudo inverse matrix is used to calculate $\mathbf{u}_{s}$ vector [11].

The block diagram of the overall UPFC control system is depicted in fig. 4. This block diagram is implemented for $\mathrm{d}-\mathrm{q}$ axis.

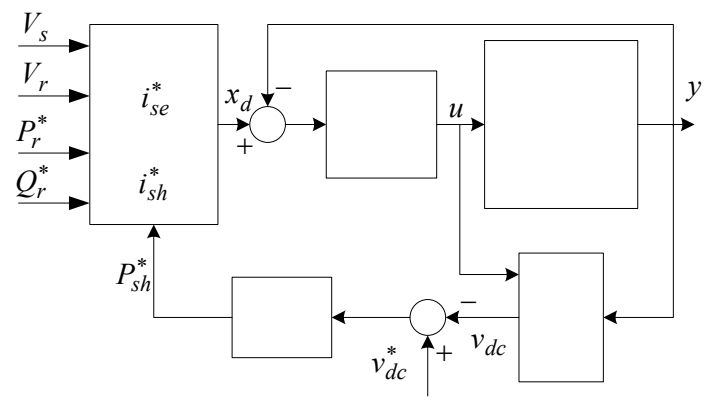

Fig 4. Block diagram of the overall UPFC control system

\section{Simulation results}

In an ideal system, there is no uncertainty in system parameters. However, in a practical system, it is considered that the system parameters are corrupted by some uncertainties. It should be mentioned that such uncertainties are usually present in any physical system and will be often 
limited to achieve the desired performance [13]. In this paper, the proposed controller is designed so as the uncertainty in the system is reduced. The uncertainty is entered to the system equations as a vector. The performance of the proposed controller, for various disturbances is evaluated through MATLAB/SIMULINK software in a two bus test system. The simulation results of proposed controller are compared with a conventional PI controller. The parameters of converters 1 and 2 of PI controllers are given in Table 1.

According to the parameters of the system and UPFC which are presented in the Table 2, the system matrices for these converters are as follow:

$$
\begin{aligned}
& A_{s e}=100 \pi\left[\begin{array}{cc}
-0.2 & 1 \\
-1 & -0.2
\end{array}\right], B_{s e}=100 \pi\left[\begin{array}{ll}
4 & 0 \\
0 & 4
\end{array}\right] \\
& A_{s h}=100 \pi\left[\begin{array}{cc}
-0.1 & 1 \\
1 & -0.1
\end{array}\right] \\
& B_{s h}=100 \pi\left[\begin{array}{cc}
6.67 & 0 \\
0 & 6.67
\end{array}\right]
\end{aligned}
$$

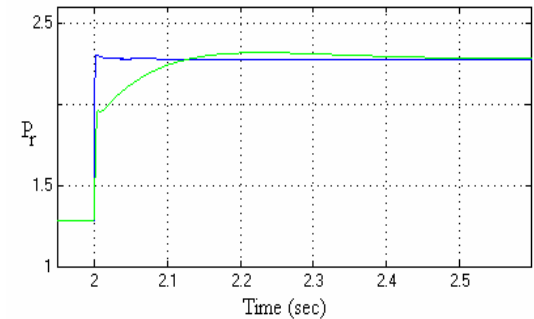

(a)

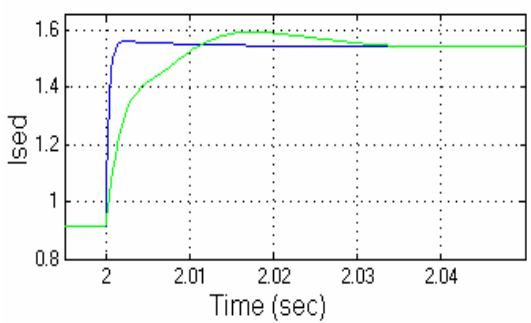

(c)

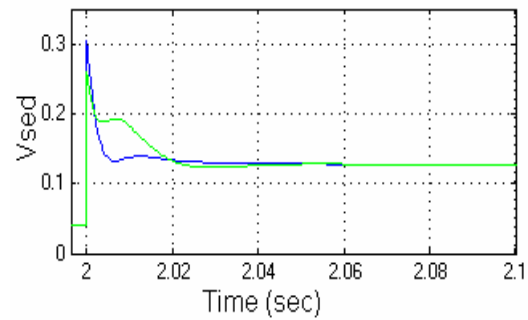

(e)
In the above matrices, fundamental frequency $\left(f_{b}\right)$ is equal to $50 \mathrm{~Hz}$. In this study, as it is shown in fig. 4, the sending and receiving end bus voltages are maintained constant and the dc link voltage, active and reactive powers of the transmission line are controlled.

Table 1: Parameters of the PI controllers

\begin{tabular}{|c|c|c|}
\hline & Converter 1 & Converter 2 \\
\hline$k_{P}$ & 0.27 & 0.3 \\
\hline$k_{I}$ & 61.3 & 65.6 \\
\hline
\end{tabular}

The initial complex power flow $\left(P_{r}+\mathrm{j} Q_{r}\right)$ at the receiving end of the transmission line is found as $(1.278-\mathrm{j} 0.5) \mathrm{pu}$. In the first case study, the active power of the transmission Line is changed from 1.278 to $2.278 \mathrm{pu}$ at $\mathrm{t}=2 \mathrm{sec}$ for a system with $10 \%$ uncertainty. The simulation results of this study are depicted in Fig. 5.

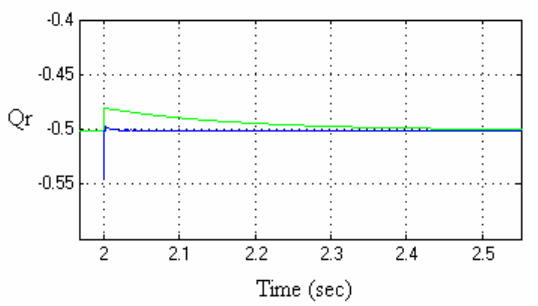

(b)

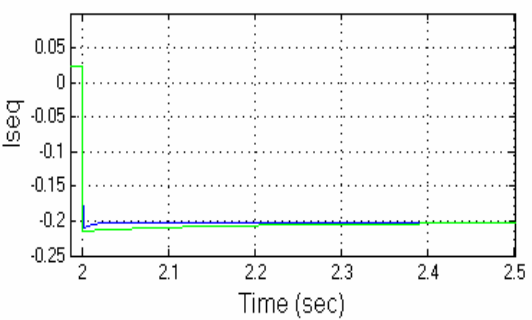

(d)

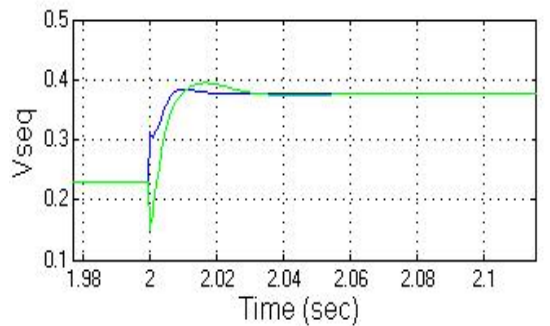

(f)

Fig. 5. Response of the UPFC system with $10 \%$ uncertainty according to the first case study Proposed controller PID controller

(a): Active power of the transmission line; (b): Reactive power of transmission line; (c): Direct axis current reference of converter 2; (d): Quadrature axis current reference of converter 2; (e): Direct axis voltage reference of converter 2; (f): Quadrature axis voltage reference of converter 2 
It is shown that the speed of response of the proposed controller is much better than that of the conventional controller approach (PI controller).

In the second case study, both the active and reactive powers of the transmission line is changed from initial values to $(2.278-\mathrm{j} 0.8)$ at $\mathrm{t}=2 \mathrm{sec}$. In this case, the uncertainty factor is considered to be equal to $15 \%$. The simulation results of this scenario are displayed in Fig. 6.

As mentioned, the reactive power of the transmission line is changed too and the uncertainty factor is changed much more than previous case, but it is seen that the proposed controller has a good response to this changes.

The active and reactive powers of the transmission line are affected strong disturbance in the latter case study. In this study which is shown in Fig. 7, the active and reactive powers are changed at $\mathrm{t}=1 \mathrm{sec}$ from $(1.278-\mathrm{j} 0.5)$ to $(2.278$ $-\mathrm{j} 0.3)$ and are changed again to initial value at $\mathrm{t}=$
$1.2 \mathrm{sec}$. In this case uncertainty factor is considered to be equal to $10 \%$.

Table 2 : Parameters of the UPFC

\begin{tabular}{|c|c|}
\hline Parameters & Value \\
\hline$R(p u)$ & 0.05 \\
\hline$\omega L(p u)$ & 0.25 \\
\hline$R_{s h}(p u)$ & 0.015 \\
\hline$\omega L_{s h}(p u)$ & 0.15 \\
\hline $1 / \omega C(p u)$ & 0.5 \\
\hline
\end{tabular}

Fig. 7 shows the response of the proposed controller for the worst case which is likely occurred. As it can be seen the proposed controller has a powerful approach to trace the system response through the uncertainty and disturbance conditions.

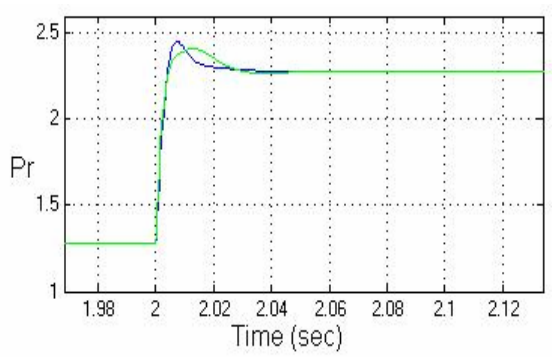

(a)

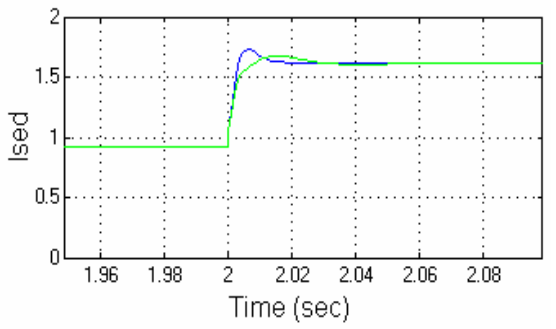

(c)

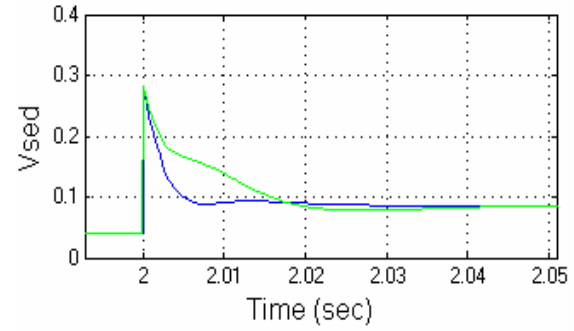

(e)

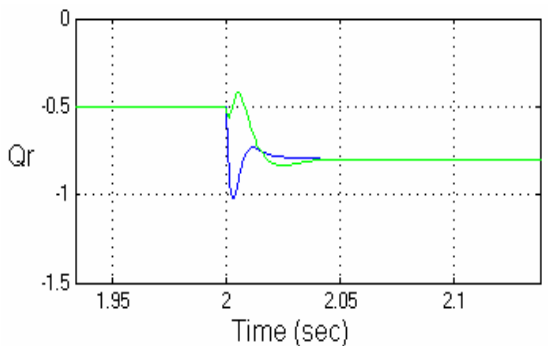

(b)

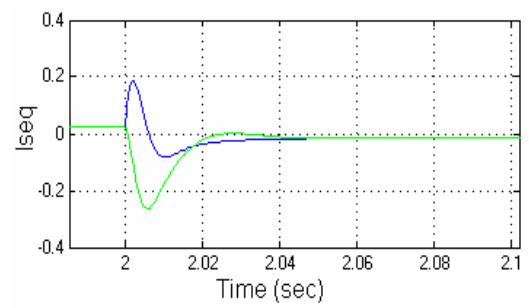

(d)

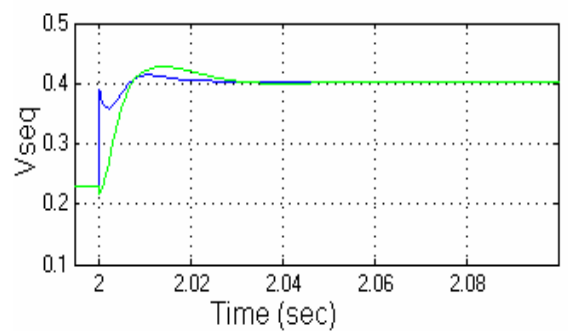

(f)

Fig. 6. Response of the UPFC system with $15 \%$ uncertainty according to the second case study Proposed controller PID controller

(a): Active power of the transmission line; (b): Reactive power of transmission line; (c): Direct axis current reference of converter 2;

(d): Quadrature axis current reference of converter 2; (e): Direct axis voltage reference of converter 2; (f): Quadrature axis voltage reference of converter 2 


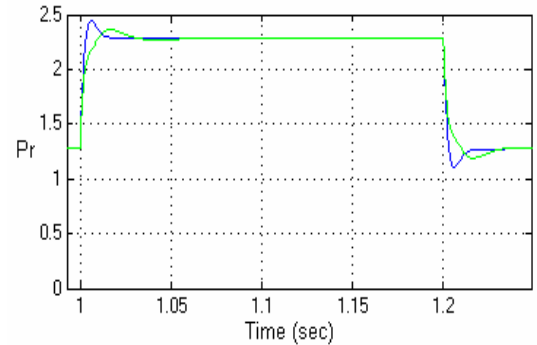

(a)

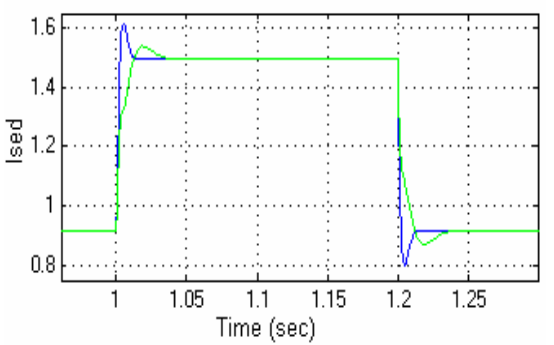

(c)

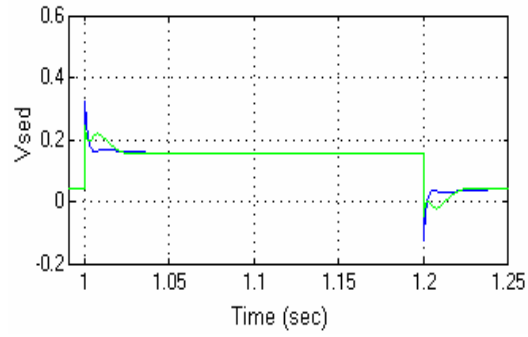

(e)

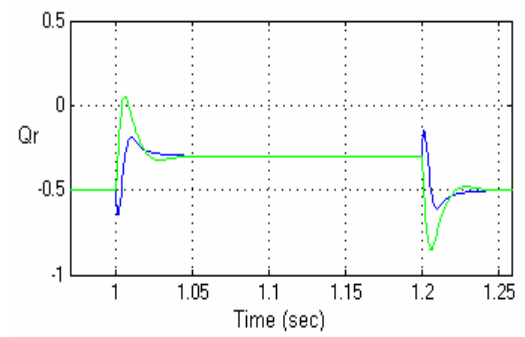

(b)

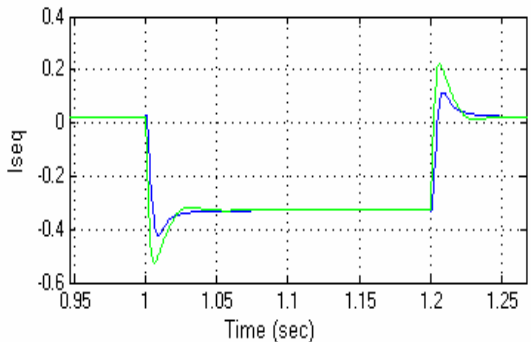

(d)

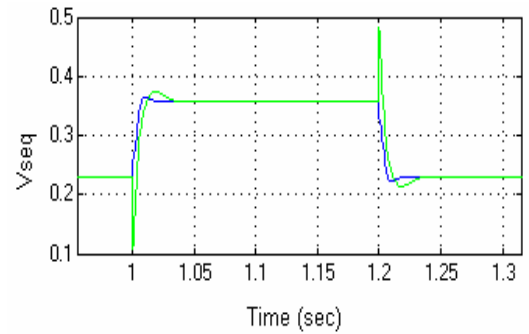

(f)

Fig. 7. Response of the UPFC system with $10 \%$ uncertainty according to the third case study Proposed controller PID controller

(a): Active power of the transmission line; (b): Reactive power of transmission line; (c): Direct axis current reference of converter 2; (d): Quadrature axis current reference of converter 2; (e): Direct axis voltage reference of converter 2; (f): Quadrature axis voltage reference of converter 2

\section{Conclusion}

The main goal of this paper is to design a controller which enables a power system to track reference signals precisely and to be robust in the presence of uncertainty of system parameters and disturbances. To reach this purpose a new controller is designed based on the Lyapunov theory. The simulation results of the proposed controller are compared with a conventional PI

\section{References}

[1] Y. H. Song and A. T. Johns, Flexible ac Transmission Systems (FACTS), The Institution of Electrical Engineers, London, 1999.

[2] L. Gyugyi, A unified power flow control concept for flexible ac transmission systems, IEE ProceedingsC 139 (4) (1992) 323-331.

[3] I. Papic, P. Zunko, D. Povh, M. Weinhold, Basic control of unified power flow controller, IEEE Trans. Power Syst. 12 (4) (1997) 1734-1739. controller. The performance of the new controller is evaluated in a two bus test system. In this study, the sending and receiving end bus voltages were maintained constant and the dc link voltage, active and reactive powers of the transmission line were controlled. The obtained results from above case studies describe the power, accuracy, fast speed and low overshoot response of the proposed controller.

[4] Q. Yu, S.D. Rund, L.E. Norum, T.M. Undeland, Dynamic control of a unified power flow controller, IEEE Proc. on PESC (1996) 508-514.

[5] I. Papic, P. Zunko, D. Povh, M. Weinhold, Basic control of unified power flow controller, IEEE Trans. Power Syst. 12 (4) (1997) 1734-1739.

[6] H. Fujita, Y. Watanabe, H. Akagi, Control and analysis of a unified power flow controller, IEEE Trans. Power Electron. 14 (6) (1999) 1021-1027.

[7] T. Makombe, N. Jenkins, Investigation of a unified power flow controller, IEE Proc. Gener. Transm. Distrib. 146 (4) (1999) 400-408. 
[8] C. Schauder and Mehta, Vector analysis and control of advanced static var compensators. IEE Proc. $-\mathrm{C}$ 140 (4) (1993) 299-306.

[9] C.M. Yam and M.H. Haque, A SVD based controller of UPFC for power flow control, Electric Power Systems Research 70 (2004) 76-84.

[10] M. I. El-Hawwary, A. L. Elshafei and et al, Output feedback control of a class of nonlinear systems using direct adaptive fuzzy controller, IEE Proc. on control theory applications 151 (5) (2004) 615-625.

[11] W. Yu, M. A. Moreno, X. Li, Observer-based neuro identifier, IEE Proc. on control theory applications 147 (2) (2000) 145-152. 